\title{
Polarografia e Métodos Polarográficos
}

\author{
MARGARIDA C. SANTOS*
}

\section{INTRODUÇÃO}

A Polarografia é um ramo dos Métodos Electroanalíticos que trata da medida e interpretação da relação existente entre a corrente e o potencial durante a electrólise de uma solução não agitada entre dois eléctrodos, um eléctrodo de referência e um micro-eléctrodo de trabalho (área de alguns $\mathrm{mm}^{2}$ ). A Polarografia é o nome particular dado ao Método Voltamétrico quando e só quando 0 eléctrodo de trabalho, ou seja aquele onde a reacção de interesse (oxidação ou redução) ocorre, é um Eléctrodo Gotejante de Mercúrio (EGM).

Aquela técnica, descoberta pelo químico checoslovaco Jaroslav Heyrovsky no início dos anos 20, (pelo que Ihe foi atribuído anos mais tarde o Prémio Nobel da Quimica) teve nas duas últimas décadas importantes desenvolvimentos, e novas técnicas polarográficas apareceram com especial destaque para os Métodos Polarográficos com Impulsos. Devido aos avanços verificados na electrónica as técnicas polarográficas mais sofisticadas podem facilmente realizar-se com equipamento disponivel comercialmente a preços muito acessiveis. Assim, a polarografia moderna é uma técnica sensivel e rápida de indiscutível interesse na maior parte das áreas de Química Analítica. Com limites de detecção da ordem dos $10^{-8} \mathrm{M}$ (caso da Polarografia de Onda Quadrada) compete nomeadamente com a Absorção Atómica na determinação de uma série de elementos (por exemplo cobre, chumbo, cádmio, zinco, niquel)

\section{ELÉCTRODO GOTEJANTE DE MERCÚRIO (EGM)}

Este tipo de eléctrodo consiste máximo, função do raio do capilar, da altura da coluna de mercúrio e da tensão superficial entre o mercúrio e a solução. Variando a altura do reservatório, variase a pressão da coluna de mercúrio e obtêm-se assim gotas reprodutíveis com tempos de vida de 1 a 6 s e diâmetros de 0,5 a $1 \mathrm{~mm}$. Como de cada vez que se forma uma gota se tem uma superfície renovada, sem substâncias adsorvidas ou depositadas, as relações corrente/potencial que se obtêm são bastante reprodutiveis, o que constitui uma vantagem sobre um metal sólido. Por exemplo num eléctrodo de Platina, os resultados são bastante dependentes das condições da superfície, nomeadamente do seu tratamento prévio.

A grande maioria das reacções estudadas com 0 eléctrodo de mercúrio são reduções 0 que é devido à janela de potencial acessivel para o mercúrio i.e. aos limites de potencial anódico e cató-

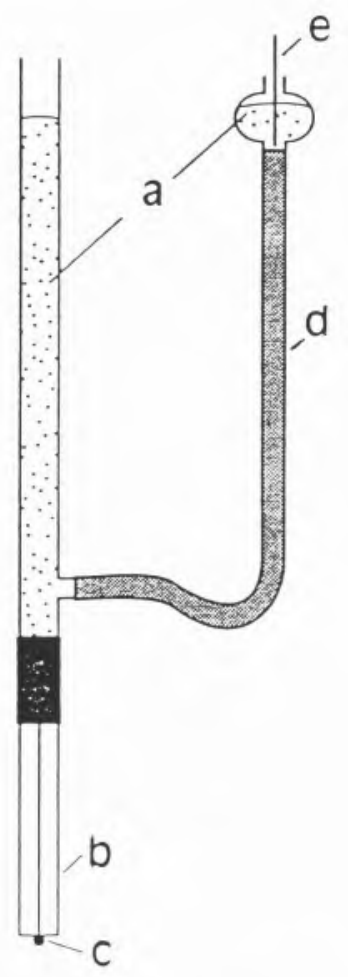

Fig. 1 - Eléctrodo gotejante de mercúrio EGM a) Mercúrio; b) Capilar; c) Gota mercúrio, d) Tubo cm tygon; e) Fio metálico dico aos quais um outro processo electroquímico pode ocorrer, mascarando o correspondente à reacção em estudo. Assim, em relação à região anódica, 0 limite é imposto pela oxidação do próprio mercúrio. 0 potencial a que esta oxidação ocorre depende da composição da solução verificando-se que num meio não complexante a reacção se dá a potenciais próximos de $0,3 \mathrm{~V}$ medidos em relação ao eléctrodo de referência saturado de calomelanos, ESC. Quanto ao limite catódico é imposto pela redução do ião $\mathrm{H}^{+}$, processo este que no mercúrio é lento estando-Ihe associado uma grande sobretensão, i.e. ocorre só a valores de potencial bastante negativos. É evidente que este valor depende da concentração hidrogeniónica sendo menos negativo quanto menor $0 \mathrm{pH}$, podendo ir até cerca $-2,0 \mathrm{~V}$ vs ESC numa solução $1 \mathrm{M}$ em NaOH . Tem-se pois que em solução aquosa a "janela de potencial" para 0 mercúrio poderá corresponder ao intervalo de potenciais compreendido entre 0,3 e -2, 0 V vs ESC.

\section{POLAROGRAFIA CLÁSSICA}

\subsection{Fundamentos}

A figura 2 mostra um polarograma típico ou seja a curva corrente (i)/potencial (E) que se obtém em polarografia para 0 caso de uma redução à superfície do eléctrodo de mercúrio numa solução não agitada, por exemplo:

$$
\mathrm{Cd}^{2+}+2 \mathrm{e} \rightleftarrows \mathrm{Cd}(\mathrm{Hg})
$$

Suponhamos que em solução existe 0 ião $\mathrm{Cd}^{2+}$ cujo potencial de decomposição, potencial ao qual se começa a verificar a redução correspondente à reacção (1), é $E_{d}$.

Para valores de potencial aplicados ao eléctrodo de mercúrio menos negativos que $E_{d}$ não ocorre reacção electroquímica, passando na célula apenas uma corrente residual de pequena intensidade. Quando o potencial aplicado atinje o valor 
de $E_{d}$ começa a notar-se um aumento de corrente correspondente à redução (1). Aumentando o potencial para valores mais negativos a concentração de iões $\mathrm{Cd}^{2+}$ à superfície do eléctrodo diminui em relação à existente no interior da solução, estabelecendo-se deste modo um gradiente de concentração. Para valores de potencial suficientemente negativos todos os iões $\mathrm{Cd}^{2+}$ que atingem a superficie do eléctrodo reduzem-se de imediato e a concentração à superfície torna-se nula. A reaç̧ão electroquímica passa a ser controlada pela velocidade à qual os iões $\mathrm{Cd}^{2+}$ vêm do interior da solução até à superfície do eléctrodo. Nestas condições um aumento do valor do potencial aplicado ao eléctrodo não conduz a um aumento de corrente, que atinge portanto um valor limite.

Qual o mecanismo responsável pelo transporte dos iões $\mathrm{Cd}^{2+}$ do interior da solução até à superfície do eléctrodo?

Estando-se a trabalhar numa solução não agitada não haverá transporte de massa por convecção. Os outros mecanismos possiveis são a migração e a difusão. Quanto ao transporte devido à migração e que é devido às atracções (ou repulsões) electrostáticas entre os iões do analito (substância a analisar) e o eléctrodo, é eliminado na presença de um electrólito de suporte, i.e., um sal cujos iões não sofrem reacção redox na zona de potencial em estudo, estando em excesso em relação ao analito e cujos iões serão os responsáveis pelo transporte da corrente no interior da solução. Deste modo o único mecanismo pelo qual os iões $\mathrm{Cd}^{2+}$ são transportados até à superfície do eléctrodo é 0 de difusão que resulta do gradiente de concentração existente. Dado que a velocidade de redução é então controlada pela velocidade de difusão, à corrente limite que se obtem, uma vez descontada a corrente residual, chama-se corrente de difusão.

Na figura 2 podem notar-se oscilações de corrente que são devidas ao crescimento e queda de cada gota de mercúrio: à medida que a gota cresce a sua área aumenta e mais substância pode alcançar a superfície para um mesmo intervalo de tempo. A corrente aumenta, portanto, até que a gota cai e então a corrente diminui drasticamente.

\section{A equação de Illkovic}

A equação fundamental que relaciona a corrente de difusão com a concentração do analito em solução é a equação de Ilkovic:

$$
\overrightarrow{i_{d}}=607 \mathrm{~nm}^{2 / 3} \mathrm{t}^{1 / 6} D^{1 / 2} \mathrm{C}
$$

Onde $\overrightarrow{i_{d}}$ representa a corrente de difusão medida na média das oscilações (Fig. 2) em Amperes, n o número de electrões trocados da reacção redox, m 0 caudal de gotejamento do mercúrio em $\mathrm{mg} / \mathrm{s}$, t 0 tempo de vida da gota em segundos, D o coeficiente de difução da espécie electroactiva em $\mathrm{cm}^{2} \mathrm{~s}^{-1}$ e C a concentração da espécie electroactiva em moles $\mathrm{cm}^{-3}$.

Na prática a corrente de difusão corresponde à diferença entre as correntes limite e residual. Entre os vários métodos possiveis para a determinação de $\overrightarrow{i_{d}}$ recomenda-se 0 método gráfico que se ilustra na fig. 3 , em que $C$ é 0 ponto médio da tangente à curva polarográfica e que une as linhas rectas ( $a$ e b) que passam pelos valores médios das correntes residual e limite. 0 valor de $\overrightarrow{\mathrm{i}}_{\mathrm{d}} \mathrm{e}$ medido na vertical e dado pelo segmento $\overline{\mathrm{AB}}$ (que passa pelo ponto $\mathrm{C}$ ). Ao valor de potencial correspondente chama-se potencial de meia onda e representa-se por $E_{1 / 2}$.

\subsection{Análise qualitativa e quantitativa}

0 valor do potencial de meia onda, $E_{1 / 2}$, assim chamado pois é o valor de potencial ao qual a corrente é igual a metade da corrente de difusão, é um valor que não depende da concentração do analito sendo uma constante num dado electrólito de suporte.

0 valor de $E_{1 / 2}$ está directamente relacionado com 0 potencial normal da reacção redox. No caso de uma reacção de eléctrodo reversivel e em que ambas a forma oxidada e a reduzida são soluvéis em solução o valor de $E_{1 / 2}$ é numericamente igual ao potencial normal da reacção redox, como acontece para os sistemas $\mathrm{Fe}^{2+} / \mathrm{Fe}^{3+}$ e quinona/quinol.

0 valor do potencial de meia onda é pois a grandeza que pode ser usada em polarografia para análise qualitativa.

Por outro lado, se se tiver em solução mais do que uma substância electroactiva a análise simultânea será possivel desde que os potenciais de meia-onda

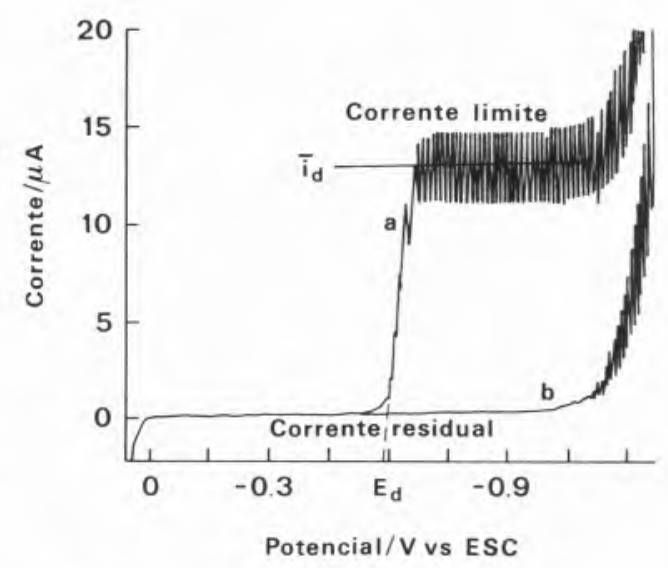

Fig. 2 - Polarogramas de a) Solução $5 \times 10^{-4} \mathrm{Mem}$ $\mathrm{Cd}^{2}+$ e $1 \mathrm{M}$ em HCl ; b) Solução $1 \mathrm{M}$ em HCl

sejam suficientemente afastados de modo a que as ondas polarográficas não se sobreponham. De um modo geral desde que $\Delta \mathrm{E}_{1 / 2}>300 / \mathrm{n} \mathrm{mV}$ a interferência é desprezável.

Como se pode ver através da equasão de llkovic (eq. 1) a amplitude da corrente de difusão depende de vários factores além da concentração do soluto. No entanto, 0 produto $\mathrm{m}^{2 / 3} \mathrm{t}^{1 / 6}$ manter-se-á constante, para um dado capilar, desde que se mantenha constante a altu$\mathrm{ra}, \mathrm{h}$, da coluna de mercúrio. Quanto ao valor do coeficiente de difusão, D, não se altera dentro dum erro de $2 \%$ desde que a temperatura não varie de mais de $1^{\circ} \mathrm{C}$. Nestas condições a equação de Ilkovic pode escrever-se na forma

$$
\overrightarrow{i_{d}}=K C
$$

ou seja a corrente de difusão é directamente proporcional à concentração da espécie electroactiva.

\section{Efeito do oxigénio na forma do polarograma}

0 oxigénio dissolvido, que existe em qualquer solução em contacto com 0 ar, reduz-se facilmente no eléctrodo de mercúrio de acordo com as reacções

$$
\begin{aligned}
& \mathrm{O}_{2}+2 \mathrm{H}^{+}+2 \mathrm{e} \rightleftarrows \mathrm{H}_{2} \mathrm{O}_{2} \quad \mathrm{E}_{1 / 2} \sim-0,1 \text { V vs ESC (2) } \\
& \mathrm{H}_{2} \mathrm{O}_{2}+2 \mathrm{H}^{+}+2 \mathrm{e} \rightleftarrows 2 \mathrm{H}_{2} \mathrm{O} \quad \mathrm{E}_{1 / 2} \sim-0,9 \text { V v ESC (3) }
\end{aligned}
$$

que dão origem a duas ondas polarográficas distintas (Fig. 4) que interferem com qualquer outra onda que possa 
ocorrer naquela região de potencial. Assim a remoção do oxigénio dissolvido é geralmente 0 primeiro passo de uma análise polarográfica e consiste em desarejar a solução durante alguns minutos ( 10 minutos) com um gás inerte (por ex. azoto).

\subsection{Limite de detecção}

0 limite de detecção em polarografia clássica corresponde a concentrações da ordem de $10^{-5} \mathrm{M}$ e é imposto pela corrente residual.

Esta corrente tem duas componentes. Uma deve-se à redução ou oxidação de impurezas inevitavelmente presentes no solvente e em qualquer electrólito. Como a concentração do electrólito de suporte é elevada esta contribuição pode ser significativa se não forem usados reagentes de grau pró-análise.

A segunda componente da corrente residual é a corrente necessária para carregar ou descarregar o condensador formado na interface mercúrio-solução e que tem a sua origem na adsorção preferencial dos iões em solução na superfície do mercúrio. Esta componente, chamada corrente capacitiva, existe sempre em qualquer experiência polarográfica independentemente da pureza dos reagentes. 0 valor desta corrente é tipicamente da mesma ordem de grandeza do da corrente de difusão devido à reaç̧ão redox de uma substância presente numa concentração de $10^{-5} \mathrm{M}$, pelo que é a principal responsável pelo valor do limite de detecção.

\section{MÉTODOS POLAROGRÁFICOS COM IMPULSOS}

As limitações da Polarografia Clássica associadas ao seu limite de detecsão são perfeitamente ultrapassadas nos Métodos com Impulsos. Desenvolvidos tendo como finalidade um menor limite de detecção, em todos os métodos com impulsos se reduz, com sucesso, a contribuição da corrente capacitiva em relação à corrente medida.

Naqueles métodos, e tal como 0 nome indica, o potencial é aplicado ao EGM periodicamente e durante curtos intervalos de tempo. A corrente em vez de ser medida durante todo o tempo de vida da gota, como acontece na polarografia clássica, é só medida durante uma fracção desse tempo (Tabela 1).

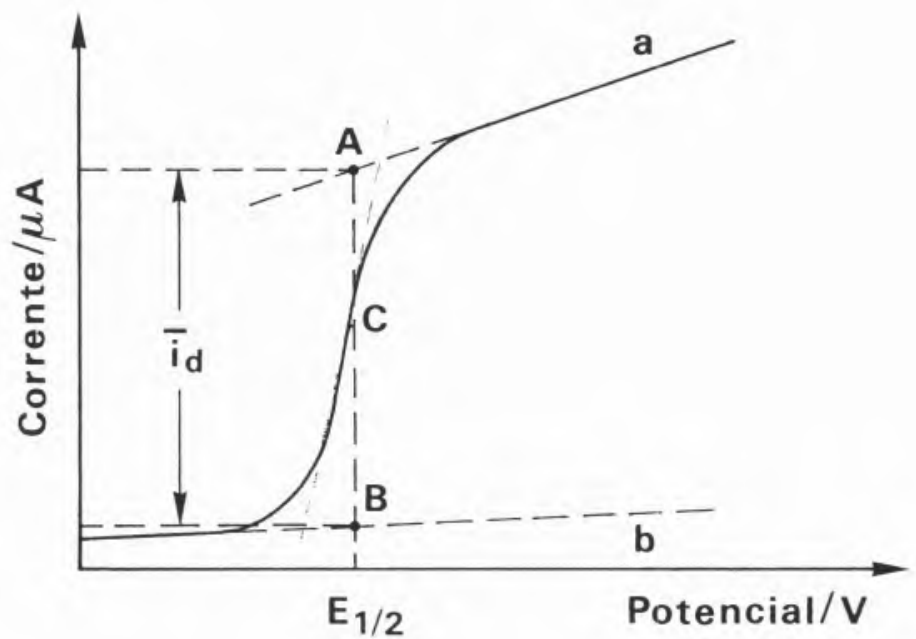

Fig. 3 - Determinação experimental de $i_{d}$ e $E_{1 / 2}$.

Durante o tempo de vida da gota $\mathrm{t}$ a corrente de difusão aumenta com $t^{1 / 6}$ como se pode ver através da equação de Ilkovic. Quanto à corrente capacitiva varia com t $\mathrm{t}^{-1 / 3}$. Deste modo medidas de corrente feitas nos últimos instantes de vida da gota maximizam a razão corrente de difusão/corrente capacitiva.

Uma compreensão mais aprofundada dos métodos com impulsos, que requer conhecimentos mais detalhados da polarografia clássica, está fora do âmbito deste texto. No entanto uma comparação dos métodos mais importantes embora que sumária parece-nos importante de modo a realçar as suas potencialidades.

Na Tabela 1 (ver pág. seguinte) apresentam-se de modo esquemático as técnicas com Impulsos mais importantes.

No que diz respeito à Polarografia Normal com Impulsos (PNI) tem-se limites de detecção da ordem de $10^{-6} \mathrm{M}$ e uma resolução igual à da Polarografia Clássica. As Polarografias Diferencial com Impulsos, PDI, e de Onda Quadrada POQ, apresentam limites de detecção entre $10^{-7}$ e $10^{-8} \mathrm{M}$. Nestas duas técnicas é possível analisar simultaneamente substâncias cujos potenciais de meia onda diferem de somente $0.05 \mathrm{~V}$.

0 aumento de resolução verificado deve-se a forma do polarograma que se obtém: um pico em vez de uma onda sigmoidal, o que é devido ao facto de se registar uma diferença de correntes por impulso como se ilustra na Tabela 1.

A Polarografia de Onda Quadrada apresenta a vantagem adicional sobre os outros métodos com impulsos de se tratar de uma técnica extraordinariamente rápida: um polarograma abrangendo uma zona de potencial de $1 \mathrm{~V}$ pode ser obtido em $0,5 \mathrm{~s}$ enquanto que 0 ensaio correspondente por Polarografia Diferencial com Impulsos demora cerca de 100 vezes mais. Nalgumas situações os polarogramas podem ser traçados na presença de oxigénio dissolvido 0 que se traduz num ganho adicional de tempo. Estes aspectos conduziram a aplicações não acessiveis aos outros métodos com impulsos, nomeadamente na detecção de compostos que emergem de um cromatógrafo.

Com os desenvolvimentos verificados na instrumentação electroquímica controlada por computadores a Polarografia de Onda Quadrada tende a tornarse no método polarográfico de excelência para análise quantitativa.

\section{APLICAÇ̃̃ES dOS MÉTODOS POLAROGRÁFICOS}

É possivel estudar por polarografia qualquer reacção electroquímica (redução ou oxidação) que se dê dentro dos limites de potencial imposto pelo siste$\mathrm{ma}$ EGM/solvente/electrólito.

Em relação a compostos inorgânicos a maior parte dos catiões metálicos reduzem-se no eléctrodo gotejante de mercúrio. Mesmo os metais alcalinos e os alcalino/terrosos são redutíveis, sendo conveniente nestas situações usar como electrólito de suporte halogenetos de tetraalquilamónio. Os métodos polarográficos são também aplicáveis na análise de aniões como o bromato, iodeto e vanadato. Uma vez que 0 ião $\mathrm{H}^{+}$par- 
ticipa nas reacções redox que envolvem aqueles aniões deve-se ter 0 meio tamporizado.

0 mesmo acontece nos processos de eléctrodo que envolvem compostos orgânicos, que tipicamente podem ser representados por

$$
\mathrm{R}+\mathrm{nH}++\mathrm{ne} \rightleftarrows \mathrm{RH}_{\mathrm{n}}
$$

onde $\mathrm{R}$ e $\mathrm{RH}_{\mathrm{n}}$ são as formas oxidada e reduzida da molécula orgânica.

Vários grupos funcionais comuns podem sofrer reacção electroquímica no EGM e logo vários compostos orgânicos podem ser determinados por métodos polarográficos. Como exemplos tem-se vitaminas como a Riboflavina, antibióticos como a Estreptomicina e esteróides como a Progesterona.

Devido aos baixos limites de detecsão requeridos em muitas destas análises os métodos com impulsos serão mais adequados.

Com o controlo cuidadoso das condições experimentais a Presição e exactidão relativa que se obtém é de 1 a $3 \%$ para concentrações da ordem de $10^{-7} \mathrm{M}$.

Esta nota sobre os métodos polarográficos ficaria incompleta sem uma referência às suas aplicações ditas não analíticas. Assim a polarografia é não só uma técnica vulgar na determinação de constantes de formação de espécies em solução como também nos permite calcular constantes de velocidade de reacções de eléctrodo e de processos químicos em solução acoplados aos processos electroquímicos.

Partilha assim com os Métodos Voltamétricos em geral a versatilidade a eles associada em áreas que não do domínio exclusivo do que se convenciona geralmente chamar da Química Analítica.

\section{BIBLIOGRAFIA}

D. A. Skoog, J.J. Leary Principles of Instrumental Analysis, 4th Ed., Saunders College Publishing, New York, 1992.

D.C. Harris Quantitative Chemical Analysis 3rd ed., W.H. Freeman and Company, New York, 1991

T. Riley, A. Watson Polarography and other Voltammetric Methods, Séries Analytical Chemistry by open Learning, John Wiley and Sons, London 1987.

A.M. Bond Modern Polarographic Methods in Analytical Chemistry, Marcel Dekker, New York, 1980.

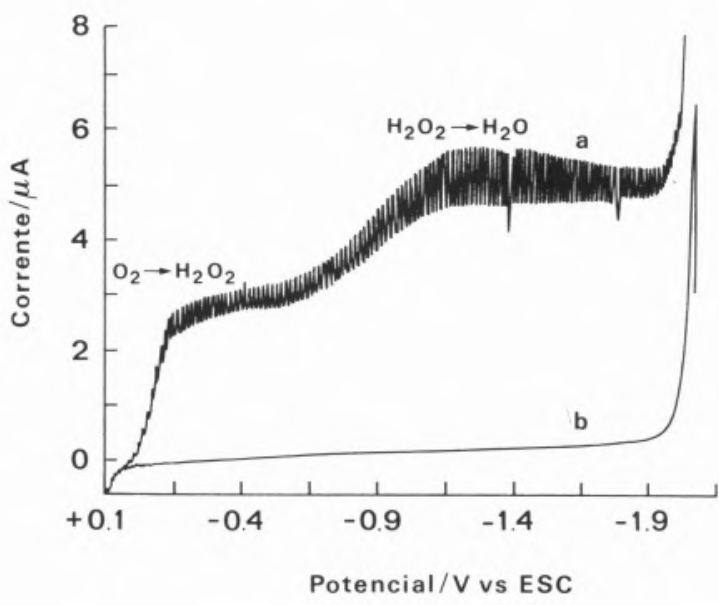

Fig. 4 - Polarogramas de uma solução $0.1 \mathrm{M} \mathrm{em} \mathrm{KCl}$ a) Saturada com ar; b) Depois de desarejamento

M.L.S.S. Gonçalves Métodos Instrumentais de Análise para Análise de Soluções, $2^{\mathrm{a}}$ ed., Fundação Calouste Gulbenkian, Lisboa, 1990.

- Centro de Química Estrutural Instituto Superior Técnico, Av. Rovisco Pais 1096 Lisboa Codex

\section{TABELA 1: TÉCNICAS POLAROGRÁFICAS COM IMPULSOS}

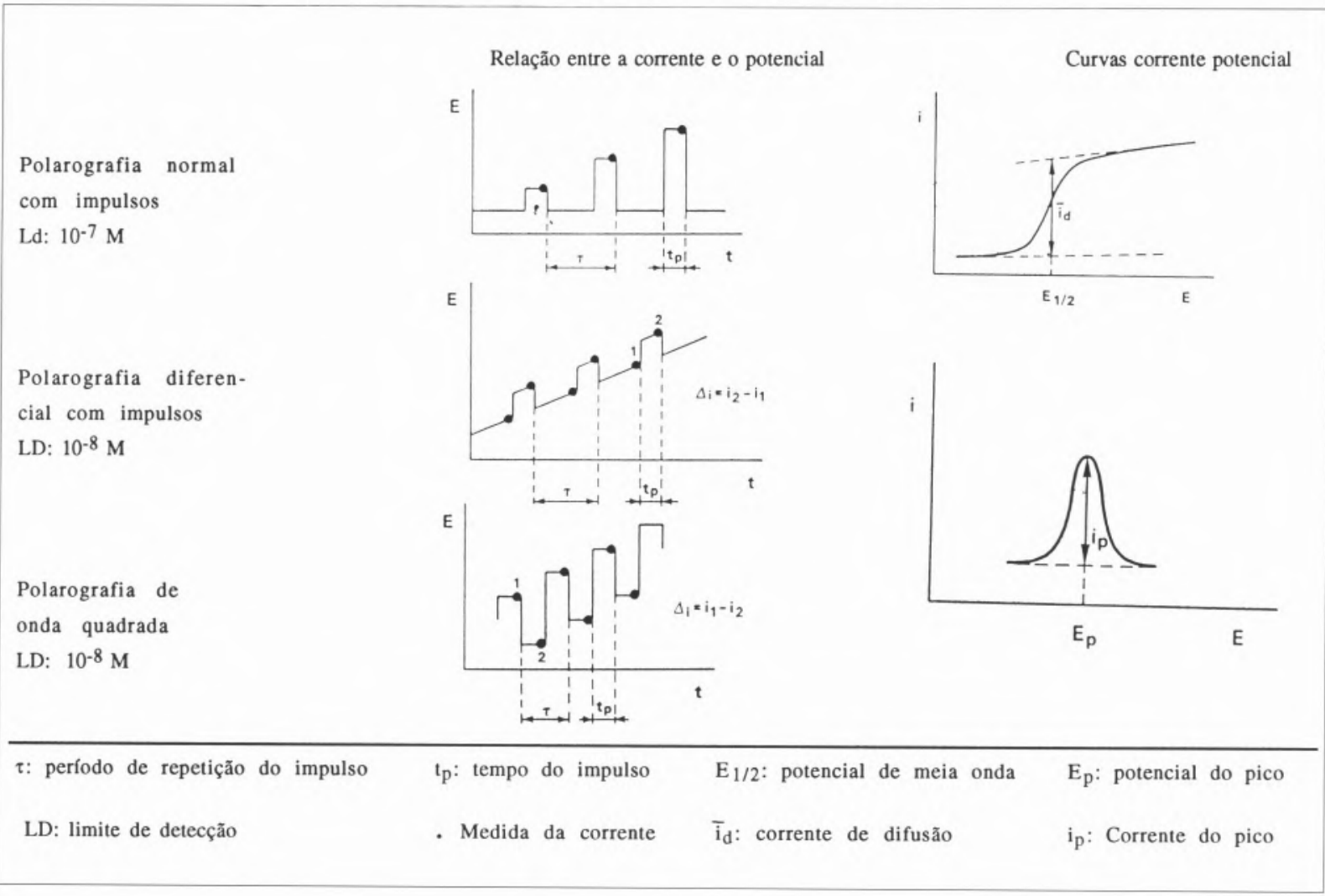

